\title{
A NEW PROOF FOR AN INEQUALITY OF JENKINS
}

\author{
GEORGE B. LEEMAN, JR.
}

Abstract. A new proof of Jenkins' inequality

$$
\operatorname{Re}\left(e^{2 i \theta} a_{3}-e^{2 i \theta} a_{2}^{2}-\tau e^{i \theta} a_{2}\right) \leqslant 1+\frac{3}{8} \tau^{2}-\frac{1}{4} \tau^{2} \log (\tau / 4), \quad 0 \leqslant \tau \leqslant 4,
$$

for univalent functions $f(z)=z+\sum_{n=2}^{\infty} a_{n} z^{n}$ is presented.

Let $S$ be the collection of functions $f(z)=z+\sum_{n=2}^{\infty} a_{n} z^{n}$ analytic and univalent in the unit disk $D$. After Löwner's [6] famous proof that $\left|a_{3}\right| \leqslant 3$ for such functions, his method was used to establish a number of theorems on the third coefficient. For example, Fekete and Szegö [1] solved a problem for odd univalent functions by examining $\left|a_{3}-\frac{1}{4} a_{2}^{2}\right|$; more generally, Goluzin [3] found the best bounds on $\left|a_{3}-\mu a_{2}^{2}\right|$ for real $\mu$, and he also maximized ||$a_{3}|-| a_{2}||$ in [2].

The most penetrating fact about the third coefficient is the spectacular inequality

(1) $\operatorname{Re}\left(e^{2 i \theta} a_{3}-e^{2 i \theta} a_{2}^{2}-\tau e^{i \theta} a_{2}\right) \leqslant 1+\frac{3}{8} \tau^{2}-\frac{1}{4} \tau^{2} \log (\tau / 4), \quad 0 \leqslant \tau \leqslant 4$,

of Jenkins [4], which includes as special cases all the results already cited (the right-hand side of (1) is defined by continuity at $\tau=0$ ).

The purpose of this paper is to show that (1) can be obtained from the Löwner theory. For each nonnegative $x$ we set

$$
\begin{aligned}
u_{x}(t) & =e^{-x} & & \text { if } 0 \leqslant t \leqslant x, \\
& =e^{-t} & & \text { if } x \leqslant t<\infty,
\end{aligned}
$$

and we prove the following

Lemma. Let $u(t)$ be continuous on $[0, \infty)$ satisfying $|u(t)| \leqslant e^{-t}$ there. If

$$
\left|\int_{0}^{\infty} u(t) d t\right|=(x+1) e^{-x}
$$

for some $x \geqslant 0$, then

$$
\int_{0}^{\infty} u(t)^{2} d t \geqslant\left(x+\frac{1}{2}\right) e^{-2 x},
$$

with equality only for $u=u_{x}$.

Proof. We claim that for each nonnegative $t$

Received by the editors November 14, 1974.

AMS (MOS) subject classifications (1970). Primary 30A34.

Key words and phrases. Univalent functions, coefficient estimates.

○ American Mathematical Society 1976 


$$
u(t)^{2}+2 e^{-x} u_{x}(t)-2 e^{-x} u(t) \geqslant u_{x}(t)^{2},
$$

with equality only for $u(t)=u_{x}(t)$. Indeed, (4) is equivalent to $\left(u(t)-e^{-x}\right)^{2}$ $\geqslant 0$ when $0 \leqslant t \leqslant x$ and to $\left[e^{-t}-u(t)\right]\left[2 e^{-x}-e^{-t}-u(t)\right] \geqslant 0$ when $x \leqslant t$ $<\infty$. By integrating both sides of (4), computing $\int_{0}^{\infty} u_{x}(t) d t$, and using (2), we arrive at (3).

Let us remark that our proof of this Lemma is based on Landau's proof of a theorem of Valiron (see (16) in [5, p. 630]); however, our hypotheses and conclusion are quite different.

According to the Löwner theory, it suffices to derive (1) for functions $f(z)=z+\sum_{n=2}^{\infty} a_{n} z^{n} \in S$ in which

$$
\begin{gathered}
a_{2}=2 \int_{0}^{\infty} k(t) e^{-t} d t \\
a_{3}=4\left(\int_{0}^{\infty} k(t) e^{-t} d t\right)^{2}-2 \int_{0}^{\infty} k(t)^{2} e^{-2 t} d t=a_{2}^{2}-2 \int_{0}^{\infty} k(t)^{2} e^{-2 t} d t,
\end{gathered}
$$

where $k(t)=e^{i \alpha(t)}$ is a continuous mapping from $[0, \infty)$ to the unit circle $\partial D$. If we set $u(t)=e^{-t} \cos \alpha(t)$, then for arbitrary $\mu \geqslant 0$, (5) and (6) yield

$$
\begin{aligned}
\operatorname{Re}\left(a_{3}-a_{2}^{2}-4 e^{-\mu} a_{2}\right) & =1-4 \int_{0}^{\infty} u(t)^{2} d t-8 e^{-\mu} \int_{0}^{\infty} u(t) d t \\
& \leqslant 1-4 \int_{0}^{\infty} u(t)^{2} d t+8 e^{-\mu}\left|\int_{0}^{\infty} u(t) d t\right|
\end{aligned}
$$

If $\operatorname{Re} a_{2}=0$, then (5) and (7) imply the sharp estimate $\operatorname{Re} a_{3} \leqslant 1$ which holds for the function $z \rightarrow z /\left(1-z^{2}\right) \in S$. Otherwise, we can find an $x \geqslant 0$ such that $\left|\int_{0}^{\infty} u(t) d t\right|=(x+1) e^{-x}$, because the range of the function $x \rightarrow(x+1) e^{-x}$ on $[0, \infty)$ is $(0,1]$. Combining (3) and (7) leads to

$$
\operatorname{Re}\left(a_{3}-a_{2}^{2}-4 e^{-\mu} a_{2}\right) \leqslant G(x)
$$

where

$$
G(x)=1-4\left(x+\frac{1}{2}\right) e^{-2 x}+8 e^{-\mu}(x+1) e^{-x} .
$$

Since $G^{\prime}(x)=8 x e^{-2 x}\left(1-e^{x-\mu}\right), G$ has a maximum at $x=\mu$, and (8) gives

$$
\operatorname{Re}\left(a_{3}-a_{2}^{2}-4 e^{-\mu} a_{2}\right) \leqslant 1+4 \mu e^{-2 \mu}+6 e^{-2 \mu} .
$$

If we replace $f(z)$ by $e^{-i \theta} f\left(e^{i \theta} z\right)$ and $\mu$ by $-\log (\tau / 4)$, then (1) follows from (9).

Because Löwner's technique is based on parametric representation of a dense subclass in $S$, we cannot determine all the cases of equality in (9) by his approach. Jenkins' method does handle this more difficult problem.

\section{REFERENCES}

1. M. Fekete and G. Szegö, Eine Bemerkung über ungerade schlichte Funktionen, J. London Math. Soc. 8 (1933), 85-89.

2. G. M. Goluzin, On distortion theorems and the coefficients of univalent functions, Mat. Sb. 1938, 321-330. (Russian)

3. - Some questions of the theory of univalent functions, Trudy Mat. Inst. Steklov. 27 (1949). (Russian) MR 13, 123. 
4. J. A. Jenkins, On certain coefficients of univalent functions, Analytic Functions, Princeton Univ. Press, Princeton, N.J., 1960, pp. 159-194. MR 22 \#126a.

5. E. Landau, Uber die Blochsche Konstante und zwei verwandte Weltkonstanten, Math. Z. 30 (1929), 608-634.

6. K. Löwner, Untersuchungen über schlichte konforme Abbildungen des Einheitskreises. I, Math. Ann. 89 (1923), 103-121.

Computer Sciences Department, IBM Thomas J. Watson Research Center, P. O. Box 218, YORKTOWN HeIGHTS, NEW YORK 10598 